\title{
Integrated spectral properties of blue concentrated star clusters of the Large Magellanic Cloud
}

\author{
J. J. Clariá ${ }^{1}$, M. C. Parisi ${ }^{1}$, A. V. Ahumada ${ }^{1}$, J. F. C. Santos Jr. ${ }^{2}$, \\ E. Bica ${ }^{3}$ and A. E. Piatti ${ }^{4}$ \\ ${ }^{1}$ Observatorio Astronómico, Universidad Nacional de Córdoba, Argentina \\ email: claria, celeste, andrea@oac.uncor.edu \\ ${ }^{2}$ Dpto. de Física, UFMG, Belo Horizonte, Brazil \\ email: jsantos@fisica.ufmg.br \\ ${ }^{3}$ Instituto de Física, UFRGS, Porto Alegre, Brazil \\ email: bica@if.ufrgs.ar \\ ${ }^{4}$ Instituto de Astronomía y Física del Espacio, Bs. As., Argentina \\ email: andres@iafe.uba.ar
}

Integrated spectra of 17 blue Large Magellanic Cloud (LMC) clusters were obtained in the (3600-6800 $\AA$ ) range using the CASLEO (Argentina) $2.15 \mathrm{~m}$ telescope. The typical resolution and dispersion were $12 \AA$ and $3.5 \AA$ pixel, respectively. Cluster ages were derived by means of two methods: the template matching, in which the observed spectra are compared and matched to template spectra with well-known determined properties, and the equivalent width (EW) method, in which diagnostic diagrams involving the sum of EWs of selected spectral lines were employed together with their calibrations with age and metallicity given by Santos \& Piatti (2004), hereafter SP. The spectra were normalized to $\mathrm{F}_{\lambda}=1$ at $\sim 5870 \AA$. The EWs of H Balmer, KCaII, G band and MgI were measured within the spectral windows defined by Bica \& Alloin (1986). We then obtained the sum of EWs of the 3 metallic lines $\left(S_{m}\right)$ and of the 3 Balmer lines $\mathrm{H} \beta, \mathrm{H} \gamma$ and $\mathrm{H} \delta$ $\left(S_{h}\right)$. As a first approach to get cluster ages, we used the diagnostic diagrams defined by SP. The clusters were then age-ranked according to the SP's calibrations. We used $S_{m}$ to get a first age estimate using: $\log \mathrm{t}(\mathrm{Gyr})=a_{0}+a_{1} S_{m}+a_{2} S_{m}^{2}$, where $a_{0}=-2.18 \pm 0.38$, $a_{1}=0.188 \pm 0.080$ and $a_{2}=-0.0030 \pm 0.0032$. We then used $S_{h}$ to get a second age estimate guided by the previous $S_{m}$ estimate, since from $S_{h}$ two solutions are possible: $\log \mathrm{t}(\mathrm{Gyr})=\left\{-b \pm\left[b^{2}-4 a\left(c-S_{h}\right)\right]^{1 / 2}\right\} / 2 a$, where $\mathrm{a}=-6.35 \pm 0.18, \mathrm{~b}=-8.56 \pm 0.35$ and $\mathrm{c}=23.32 \pm 0.20$. The average of these two estimates is listed in column 7 of Table 1 .

All 17 clusters are well represented by blue stellar populations, according to their spectral properties. Since the continuum distribution is affected by reddening, we firstly adopted a colour excess $\mathrm{E}(\mathrm{B}-\mathrm{V})$ for each cluster, taking into account the Burstein \& Heiles (1982) extinction maps. Secondly, we corrected the observed spectra accordingly and then we applied the template matching method. The resulting ages, together with estimates from the literature (whenever available), were used to get final averaged ages (Table 1). Piatti, Bica, Geisler et al. (2003a) observed in the Washington system 6 LMC clusters, which increased up to 37 the total sample of clusters with uniform estimates of age and metallicity. The general tendency is for the older clusters to lie in the outer disk regions of the galaxy while the younger ones tend to be located not far from or in the bar. This tendency is compatible with the findings of Smecker-Hane, Cole, Gallagher et al. (2002), who derived the LMC star formation history from HST observations of field stars. 
Table 1. Cluster parameters

\begin{tabular}{lcccccc}
\hline Cluster & $E(B-V)$ & $\begin{array}{c}t_{\text {literature }} \\
(\mathrm{Gyr})\end{array}$ & Ref. & $\begin{array}{c}t_{\text {Sh,Sm }} \\
(\mathrm{Gyr})\end{array}$ & $\begin{array}{c}t_{\text {template }} \\
(\mathrm{Gyr})\end{array}$ & $\begin{array}{c}t_{\text {adopted }} \\
(\mathrm{Gyr})\end{array}$ \\
\hline NGC 1804 & 0.08 & $0.08 \pm 0.01$ & 1 & $0.035 \pm 0.004$ & $0.05 \pm 0.01$ & $0.06 \pm 0.02$ \\
NGC 1839 & 0.06 & $0.10 \pm 0.01$ & 1 & $0.09 \pm 0.02$ & 0.06 & $0.09 \pm 0.03$ \\
& & $0.033 \pm 0.008$ & 2 & & & \\
SL 237 & 0.07 & $0.125 \pm 0.025$ & 4 & & & \\
& & $0.038 \pm 0.004$ & 1 & $0.03 \pm 0.02$ & $0.05 \pm 0.01$ & $0.04 \pm 0.02$ \\
NGC 1870 & 0.08 & $0.09 \pm 0.01$ & 1 & $0.033 \pm 0.004$ & $0.05 \pm 0.01$ & $0.06 \pm 0.03$ \\
& & $0.07 \pm 0.03$ & 2 & & & \\
NGC 1894 & 0.09 & $0.071 \pm 0.008$ & 1 & $0.10 \pm 0.08$ & $0.13 \pm 0.03$ & $0.10 \pm 0.03$ \\
NGC 1902 & 0.04 & - & & $0.07 \pm 0.03$ & 0.06 & $0.07 \pm 0.03$ \\
NGC 1913 & 0.09 & $0.024 \pm 0.002$ & 1 & $0.03 \pm 0.02$ & 0.06 & $0.04 \pm 0.02$ \\
NGC 1932 & 0.05 & - & & $0.2 \pm 0.1$ & $0.4 \pm 0.2$ & $0.3 \pm 0.2$ \\
NGC 1943 & 0.08 & $0.14 \pm 0.02$ & 1 & $0.08 \pm 0.06$ & $0.28 \pm 0.08$ & $0.14 \pm 0.06$ \\
& & $0.10 \pm 0.01$ & 3 & & & \\
NGC 1940 & 0.06 & - & 1 & $0.06 \pm 0.02$ & 0.06 & $0.06 \pm 0.02$ \\
NGC 1971 & 0.06 & $0.10 \pm 0.01$ & 1 & $0.06 \pm 0.04$ & $0.05 \pm 0.01$ & $0.06 \pm 0.02$ \\
SL 508 & 0.06 & $0.10 \pm 0.01$ & 1 & $0.039 \pm 0.008$ & 0.06 & $0.06 \pm 0.04$ \\
NGC 2038 & 0.06 & $0.13 \pm 0.02$ & & $0.3 \pm 0.2$ & $0.13 \pm 0.03$ & $0.08 \pm 0.05$ \\
SL 709 & 0.06 & - & & $0.05 \pm 0.02$ & 0.06 & $0.05 \pm 0.02$ \\
NGC 2118 & 0.07 & - & & $0.03 \pm 0.02$ & 0.06 & $0.04 \pm 0.02$ \\
NGC 2130 & 0.05 & - & & $0.085 \pm 0.008$ & $0.05 \pm 0.01$ & $0.07 \pm 0.02$ \\
NGC 2135 & 0.05 & - &
\end{tabular}

References: (1) Pietrzyński \& Udalski (2000); (2) Alcaino \& Liller (1987); (3) Bono, Marconi, Cassisi et al. (2005); (4) Piatti, Bica, Geisler et al. (2003b).

We used the integrated UBV colours of 624 LMC clusters and associations obtained by Bica, Clariá, Dottori et al. (1996) to check the trend of (U-B) and (B-V) with age according to the present estimates. The colour gap seen in both $(\mathrm{U}-\mathrm{B})$ and $(\mathrm{B}-\mathrm{V})$ is a real feature first identified by van den Bergh (1981). The gap is probably a natural consequence of cluster evolution with increasing metallicities towards the present, and epochs of reduced cluster formation between $\approx 300 \mathrm{Myr}$ and $\approx 1$ Gyr. At least in the LMC bar, such a period of reduced cluster formation is not observed for the field stars (Smecker-Hane, Cole, Gallagher et al. 2002).

Keywords. galaxies: star clusters - Magellanic Clouds - techniques: spectroscopy

\section{References}

Alcaino, G. \& Liller, W. 1987, AJ 94, 372

Bica, E. \& Alloin, D. 1986, A\&A 162, 21

Bica, E., Clariá, J.J., Dottori, H., Santos Jr., J.F.C. \& Piatti, A.E. 1996, ApJS 102, 57

Bono, G., Marconi, M., Cassisi, S., et al. 1996, ApJS 102, 57

Burstein, D. \& Heiles, C. 1982, AJ 87, 1165

Piatti, A.E., Bica, E., Geisler, D. \& Clariá, J.J. 2003a, MNRAS 344, 965

Piatti, A.E., Bica, E., Geisler, D. \& Clariá, J.J. 2003b, MNRAS 343, 841

Pietrzyński, G. \& Udalski, A. $2000 A c A$ 50, 337

Santos Jr., J.F.C. \& Piatti, A.E. 2004, A\&A 428, 79 (SP)

Smecker-Hane, T.A., Cole, A.A., Gallagher, J.S. \& Stetson, P.B. 2002 ApJ 566, 239

van den Bergh, S. 1981, A\&AS 46, 79 